\title{
Ecuaciones alométricas para estimar tasas de crecimiento de Myroxylon balsamum, Minquartia guianensis y Otoba parvifolia en la Amazonía ecuatoriana
}

\section{Allometric Equations for Estimating Myroxylon Balsamum, Minquartia Guianensis, and Otoba Parvifolia Growth Rates in Ecuadorian Amazonia}

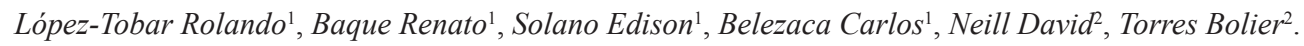

${ }^{I}$ Docente Investigador - Universidad Técnica Estatal de Quevedo UTEQ

${ }^{2}$ Docente Investigador - Universidad Estatal Amazónica UEA

Rec.: 18.12.2017. Acept.: 30.04.2018 Publicado el 2 de Julio de 2018

\section{Resumen}

T a evaluación del análisis de las tasas de crecimiento de Llas especies forestales Myroxylon balsamum (L.) Harms (Bálsamo) y Minquartia guianensis Aubl. (Guayacán negro) y Otoba parvifolia (Doncel), establecidas en rodales de investigación en la Estación Científica Jatun Sacha (ECJS), con una edad de 20 años, fue el motivo principal de esta investigación. De acuerdo a los resultados obtenidos se encontró que el Bálsamo necesita 59 años para alcanzar los 60 centímetros de Diámetro Mínimo de Corta (DMC), que está determinado por el Acuerdo Ministerial 039 del Ministerio del Ambiente, el Guayacán negro Huambula, necesita 69 años para llegar a los 40 centímetros de diámetro, mientras que el Doncel necesita 27 años para obtener $50 \mathrm{~cm}$ de diámetro.

Palabras claves: Tasas de crecimiento, Rodales de investigación, Jatun Sacha, Diámetro Mínimo de Corta
E valuating the growth rates of the forest species Myroxylon balsamum (L.) Harms (Balsamo), Minquartia guianensis Aubl. (Guayacan negro), and Otoba parvifolia (Doncel), established in research stands at the Jatun Sacha Science Station (JSSS) at the age of 20, was the main reason for this research. According to the results obtained, it was found that Balsamo needs 59 years to reach the 60 centimeter Minimum Cutting Diameter (DMC), established by the Resolution 039 of the Ministry of the Environment; Guayacán Negro Huambula needs 69 years to attain the 40 centimeter diameter, whereas Doncel needs 27 years to obtain $50 \mathrm{~cm}$ of diameter.

Keywords: Growth Rates, Research Rods, Jatun Sacha, Minimum Cutting Diameter. 


\section{Introducción}

E cuador, en las últimas décadas, ha ganado fama a nivel mundial por su alta diversidad biológica y está incluido en la lista de los 17 países "megadiversos" (Neill et al., 1993; Gentry, 1996; Neil, 2012). Una de las regiones más diversas y ricas del país es la región amazónica ecuatoriana, donde existen unas 2.000 especies de plantas, de las cuales 1.356 se encuentran por debajo de los 500 msnm (Cerón, 1993a; Jorgensen \& León-Yánez, 1999; Guevara et al., 2009). En esta región en áreas relativamente pequeñas (1 ha) se puede encontrar cifras altas de especies: ECJS 250 especies, Payamino 243 especies, Cuyabeno 307 especies, Añangu 228 especies de árboles mayores a $10 \mathrm{~cm}$ de diámetro medido a $1.30 \mathrm{~m}$ desde la superficie del suelo, (Revelo \& Palacios, 2005). Sin embargo, estos ecosistemas son altamente diversos, heterogéneos y se han visto severamente alterados, especialmente por actividades como cambio de uso del suelo extracción selectiva de madera (Pichon et al., 1999; Pan et al., 2004; Torres et al., 2013).

Los datos de deforestación actuales provienen de la estimación de la tasa de deforestación del Ecuador Continental, presentados por el Ministerio del Ambiente, 2012, reportan que la tasa anual de cambio de cobertura boscosa en el Ecuador continental es de $-0.71 \%$ para el período 1990 - 2000 y de $-0.66 \%$ para el período 2000 - 2008. Esto corresponde a una deforestación anual promedio de 89.944 ha/año y 77.647 ha/año para ambos períodos, respectivamente. En este proceso de desforestación se pierden muchas especies de alto valor ecológico y comercial como Myroxylon balsamum (L.) Harms (Balsamo), Minquartia guianensis Aubl. (Guayacán negro) y Otoba parvifolia (Markgr.) A.H. Gentry (Doncel) entre otras (López, 2014a).

Por lo tanto es, necesario realizar investigaciones sobre las tasas de crecimientos de especies forestales de valor comercial para determinar en cuantos años se puede lograr el Diámetro Mínimo de Corta (DMC) para su aprovechamiento, que esta determinado por el Acuerdo Ministerial 0125 del Ministerio del Ambiente (MAE, 2015). De acuerdo a este documento, los DMC de las especies Myroxylon balsamum, Minquartia guianensis y Otoba parvifolia para la Región Amazónica y estribaciones son: 60,40 y 50 centímetros de diámetro respectivamente, (MAE, 2004; MAE, 2009; MAE, 2010).

En este marco, el presente trabajo representa un esfuerzo por aportar datos sobre los niveles de crecimiento de estas tres especies forestales mencionadas en la provincia de Napo, Amazonia ecuatoriana.

Materiales y métodos

\section{Sitio de estudio}

Las tres especies forestales investigadas Myroxylon balsamum (L.) Harms (Balsamo) y Minquartia guianensis Aubl. (Guayacán negro) y Otoba parvifolia (Markgr.) A.H. Gentry (Doncel), corresponden rodales de investigación establecidos por la ECJS (Figura 1) en el año 1991.
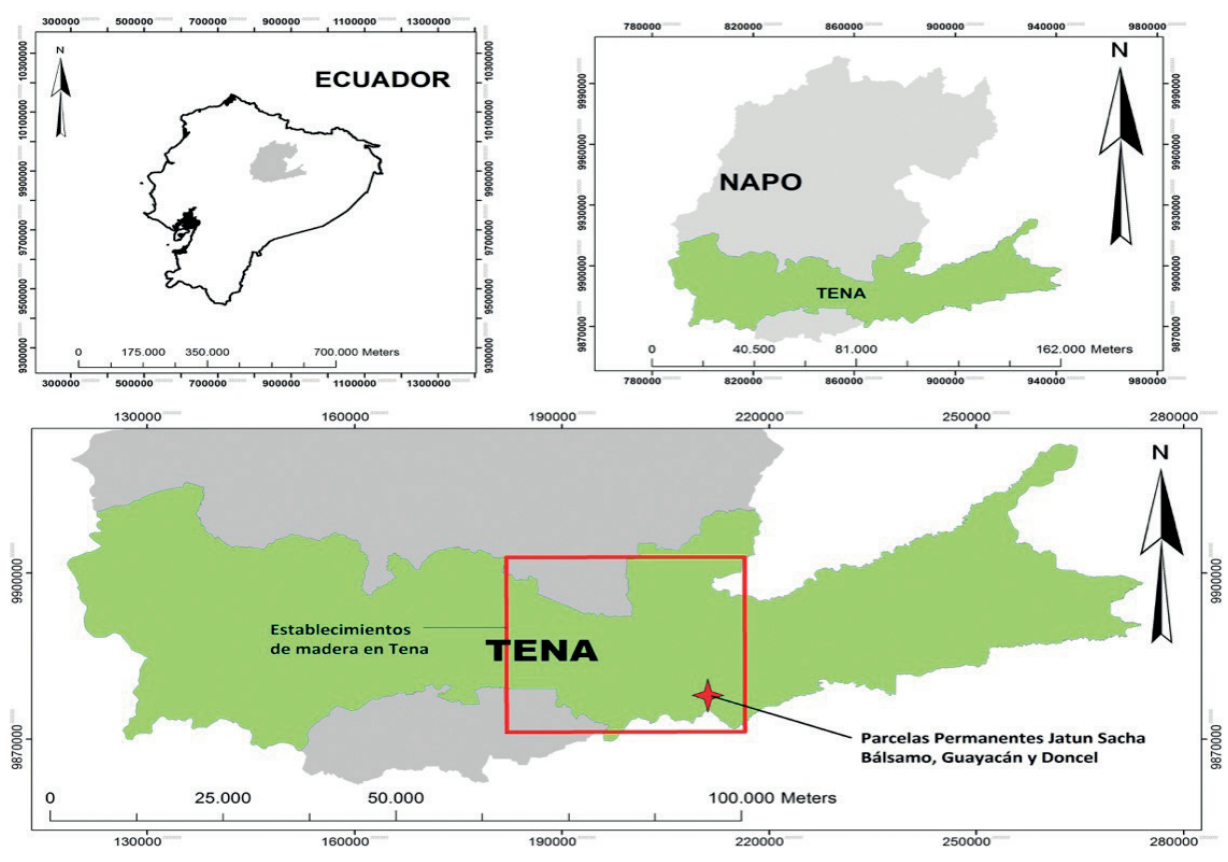

Figura 1. Ubicación de la zona de estudio, cantón Tena, provincia de Napo Fuente: Unidad SIG - UEA, Puyo (2013). 


\section{Principales características de las especies estudiadas}

Las tres especies estudiadas son nativas de la Amazonía ecuatoriana (Cuadro 1) e importantes por sus valores ecológicos y comerciales, las principales características son descritas de información secundaria:

Cuadro 1. Características de la zona de estudio, ECJS, Napo, Ecuador.

\begin{tabular}{ll}
\hline Variables & \\
\hline Temperatura & $25^{\circ} \mathrm{C}$ \\
Precipitación & $3673 \mathrm{~mm}$ \\
Humedad relativa & $90 \%$ \\
Elevación & $450 \mathrm{msnm}$ \\
Pendiente & Colinas medianamente disectadas \\
Características del suelo: & Franca profundidad $25 \mathrm{~cm}$. \\
$\quad$ Textura & Poco permeables, mal drenados \\
Drenaje & Baja \\
Fertilidad & Acido 5.1 \\
pH & Rastrojos derivados de cultivos abandonados \\
Uso anterior & Según Holdridge bHT - Bosque Húmedo Tropical \\
Formación ecológica &
\end{tabular}

Fuente: Libro Revelo, N. \& Palacios, W. 2005. Avances Silviculturales en la Amazonia ecuatoriana.

\section{Bálsamo Myroxylon balsamum}

Árbol de hasta 40 metros o más de altura, copa por lo general pequeña y estrecha con relación a la altura total del árbol, hojas pinnadamente compuestas y alternas de tamaño mediano entre 10 y $15 \mathrm{~cm}$ de largo con 7 a 15 foliolos alternos a lo largo del raquis delgado con una notable forma de zigzag (Palacios, 2011). Foliolos de color verde lustroso y por laminas con los bordes marcadamente undados, osea con curvas u ondas como una lámina corrugada para techo, flores pequeñas con 5 pétalos flores con muchos estambres poseen aroma dulce inflorescencias con ramos erectos de hasta $20 \mathrm{~cm}$ de largo (Melo \& Vargas, 2002). De sus frutos y hojas se extraen aceites esenciales aromáticos, además, al igual que la corteza se los utiliza para remedios caseros contra el catarro, laringitis, bronquitis y en infusión para el tratamiento de la menstruación abundante (López, 2014b). La madera se caracteriza por ser dura y resistente al intemperie, color castaño rojizo (Limogni \& Guiracocha, 2012).

\section{Guayacán Minquartia guianensis}

Árbol de hasta 30 metros o más de altura, raíces tablares poco desarrolladas. Corteza externa parda con grietas superficiales, la corteza interna es crema - oscura a crema - parda, muy fibrosa. Hojas simples, alternas helicoidales, oblongas; envés parduzco; peciolos $3-5 \mathrm{~cm}$ de largo, curvados en el ápice; nervios secundarios paralelos entre sí, numerosos. Inflorescencia en racimo, hasta $15 \mathrm{~cm}$ de largo (Plumptre, 1995). Fruto es una drupa oblonga, $3 \mathrm{~cm}$ de largo de una semilla, presencia de látex blanco en ramitas terminales y peciolos (Revelo \& Palacios 2005). Se distribuye desde América central hasta la cuenca amazónica en el bosque húmedo tropical primario. Es una especie rara y una típica esciófita (Janzen et al., 1990). La madera de alta densidad es muy resistente a la humedad, por lo que se usa para pilotes de casas en el sector rural. Se dice que las vigas de las casas duran hasta 30 años y más en pleno contacto con la humedad. La corteza se utiliza tradicionalmente como desparasitante (López, 2014c).

\section{Doncel Otoba parvifolia}

Árbol de hasta 30 metros de altura y 80 centímetros de DAP con raíces tablares poco desarrolladas, corteza exterior parda muy suave desprendible, corteza interna rojiza - rosada, vierte abundante sabia roja - oscura, hojas simples, alternas dísticas oblongas, inflorescencia en racimo zigzagueante de hasta $10 \mathrm{~cm}$ de largo flores es fascículos de $3 \mathrm{~mm}$ de largo, pardas unisexuales con tres tépalos (Jørgensen \& León-Yánez, 1999; Cerón et al., 2005b). La semilla es una drupa dehiscente con pericarpio de más o menos $4 \mathrm{~mm}$ de espesor, semilla globosa o subglobosa cubierta por un arilo crema y laciniado; la madera se usa para contrachapados molduras y otros, es muy usado en la construcción (López, 2014a)

\section{Tratamiento de los datos y análisis estadísticos}

$\mathrm{U}$ na vez levantados los datos de todos los individuos presentes en las parcelas permanentes con el inventario total, se ordenó en el software de Microsoft Excel, donde se procedió a determinar el área basal $(\mathrm{G})$ y el volumen total con las fórmulas a continuación descritas:

o Área Basal (G)

Donde: 
$\mathbf{G}=$ Área basal (m2)

DAP $=$ Diámetro $(\mathrm{m})$

o Volumen total (Vt)

$\mathbf{V t}=\mathrm{G} \times \mathrm{xh} \times \mathrm{f}$

Donde:

$\mathbf{V t}=$ Volumen total del árbol

$\mathbf{G}=$ Área basal

$\mathbf{h t}=$ Altura total

$\mathbf{f}=$ Factor de forma $(0,7$ para especies latifoliadas $)$

Seguidamente en función de las sumatorias de árboles/ parcelas, área basal/parcelas y volumen/parcelas, utilizando el mismo software de Microsoft Excel se calcularon varios parámetros estadísticos entre ellos: promedios, varianzas, desviaciones estándar y coeficientes de variación; cuyos promedios y desviaciones se utilizaron para la validación estadística de la hipótesis de la investigación.

\section{Ecuaciones alométricas}

El desarrollo de simuladores de crecimiento y rendimiento forestal basados en modelos de rodal, modelos de distribuciones diamétricas y modelos de árbol individual es una respuesta a los cambios actuales en los objetivos del manejo (Cuadro 2). Son también una respuesta a los cambios en la disponibilidad, necesidades y flujo de información en la práctica forestal (Pretzsch et al., 2006).

En la actualidad, la descripción de la dinámica forestal requiere información adicional a los atributos comunes de árboles y de rodales, tales como características financieras y de rendimiento y crecimiento, o variables estructurales, económicas y sociales, las cuales han ganado preponderancia en el manejo moderno de ecosistemas y que están siendo tomadas en cuenta en la simulación del crecimiento forestal. Esta información combinada con otros componentes, por ejemplo, hardware y software especializado, han hecho posible la introducción de los simuladores de crecimiento en la práctica forestal. (Vargas et al., 2008)

Cuadro 2. Ecuaciones Alometricas aplicadas en los datos tomados de las parcelas permanentes establecidas en la EBJS, provincia Napo

\begin{tabular}{ll}
\hline Modelo & Ecuación \\
\hline Mathcad & $\mathrm{M}=\mathrm{Co}(1+\mathrm{r}) \mathrm{n}$ \\
Von Bertalanffy & $\mathrm{Y}=\mathrm{a} *(1-\mathrm{e}-\mathrm{bt}) 3$ \\
Schumacher & $\mathrm{Y}=\mathrm{a} * \mathrm{e}\left(-\mathrm{bt} \mathrm{t}^{\wedge}(-1)\right)$ \\
Yoshida & $\mathrm{Y}=\mathrm{a}^{*} \mathrm{td} /(\mathrm{b}+\mathrm{td})+\mathrm{c}$ \\
Chapman-Richards & $\mathrm{Y}=\mathrm{a}^{*}(1-\mathrm{e}(-\mathrm{bt})) \mathrm{c}$ \\
Logístico & $\mathrm{Y}=\mathrm{a} /\left(1+\mathrm{b}^{*} \mathrm{e}(-\mathrm{ct})\right)$ \\
Gompertz & $\mathrm{Y}=\mathrm{a}^{*} \mathrm{e}-\mathrm{b}^{*} \mathrm{e}(-\mathrm{ct})$ \\
Weibull & $\mathrm{Y}=\mathrm{a}^{*}(1-\mathrm{e})-(\mathrm{bt}) \mathrm{c}$ \\
Exponencial & $\mathrm{Y}=\mathrm{a}^{*} \mathrm{e}(\mathrm{bt})$ \\
\hline
\end{tabular}

\section{Resultados y Discusiones}

\section{Incremento diámetro altura}

$\mathrm{Z}^{1}$ promedio de área basal por hectárea, determinado para la - parcela de Myroxylon balsamum, fue de 0.03 para el año 1993; 0.61 para el año 2002 y 1.43 metros cuadrados para el año 2013, mientras que el volumen promedio por hectarea en esos mismos años fue de $0.08 ; 5.17$ y 18.01 metros cúbicos. El promedio de área basal por hectárea, determinado para la parcela de Minquartia guianensis, fue de 0.01 para el año 1993; 0,08 para el año 2002 y 0,33 metros cuadrados para el año 2013, mientras que el volumen promedio por hectárea en esos mismos años fue de 0,$00 ; 0,03$ y 2,26 metros cúbicos. El promedio de área basal por hectárea, determinado para la parcela de Otoba parvifolia, fue de 0.28 para el año 1993; 0.63 para el año 2002 y 2,86 metros cuadrados para el año 2013, mientras que el volumen promedio por hectárea en esos mismos años fue de 0,$018 ; 0,1669$ y 39,83 metros cúbicos (Cuadro 3). 
Cuadro 3. Registro de la toma de datos en parcelas de Myroxylon balsamum (L.) Harms (Bálsamo), Minquartia guianensis Aubl. (Guayacán negro) y Otoba parvifolia (Doncel) durante los años 1993-2013 en la Estación Biológica Jatun Sacha, Napo, 2013

\begin{tabular}{|c|c|c|c|c|c|c|c|c|c|}
\hline Parcela & $\begin{array}{l}\text { No. } \\
\text { Árboles }\end{array}$ & $\begin{array}{c}\text { Año } \\
\text { toma } \\
\text { datos }\end{array}$ & $\begin{array}{c}\text { Prom } \\
\text { Diametro } \\
(\mathrm{cm})\end{array}$ & $\begin{array}{c}\text { IMA } \\
\text { Diametro } \\
(\mathrm{cm})\end{array}$ & $\begin{array}{c}\text { Prom. } \\
\text { Altura } \\
\text { (m) }\end{array}$ & $\begin{array}{c}\text { IMA } \\
\text { Altura } \\
\text { (cm) }\end{array}$ & $\begin{array}{c}\text { Prom } \\
\text { Área } \\
\text { basal } \\
(\mathrm{m} 2)\end{array}$ & $\begin{array}{c}\text { Prom } \\
\text { Vol. } \\
\text { (m3) }\end{array}$ & $\begin{array}{c}\text { Sobrev } \\
\%\end{array}$ \\
\hline Balsamo & 156 & 1991 & \multicolumn{7}{|c|}{ Establecimiento } \\
\hline Balsamo & 100 & 1993 & 2,0 & 0,75 & 2,4 & 1,2 & 0,03 & 0,08 & 64 \\
\hline Balsamo & 78 & 2002 & 8,8 & 0,84 & 8,5 & 0,81 & 0,61 & 5,17 & 50 \\
\hline Balsamo & 70 & 2013 & 13,49 & 0,85 & 12,6 & 0,81 & 1,43 & 18,01 & 45 \\
\hline Guayacán & 130 & 1991 & \multicolumn{7}{|c|}{ Establecimiento } \\
\hline Guayacán & 130 & 1993 & 1,00 & 0,02 & 0,20 & 0,30 & 0,01 & 0,00 & 100 \\
\hline Guayacán & 91 & 2002 & 3,1 & 0,3 & 0,46 & 0,41 & 0,08 & 0,03 & 70 \\
\hline Guayacán & 83 & 2013 & 6,5 & 0,52 & 6,81 & 0,93 & 0,33 & 2,26 & 64 \\
\hline Doncel & 20 & 1991 & \multicolumn{7}{|c|}{ Establecimiento } \\
\hline Doncel & 20 & 1993 & 0,60 & 0,91 & 0,67 & 0,63 & 0,28 & 0,018 & \\
\hline Doncel & 18 & 2002 & 2,85 & 0,79 & 2,62 & 0,74 & 0,63 & 0,16 & \\
\hline Doncel & 18 & 2013 & 19,11 & 0,85 & 13,89 & 0,81 & 2,86 & 39,83 & \\
\hline
\end{tabular}

Fuente: Datos colectados en los años 1993 y 2002 (Revelo \& Palacios, 2005)

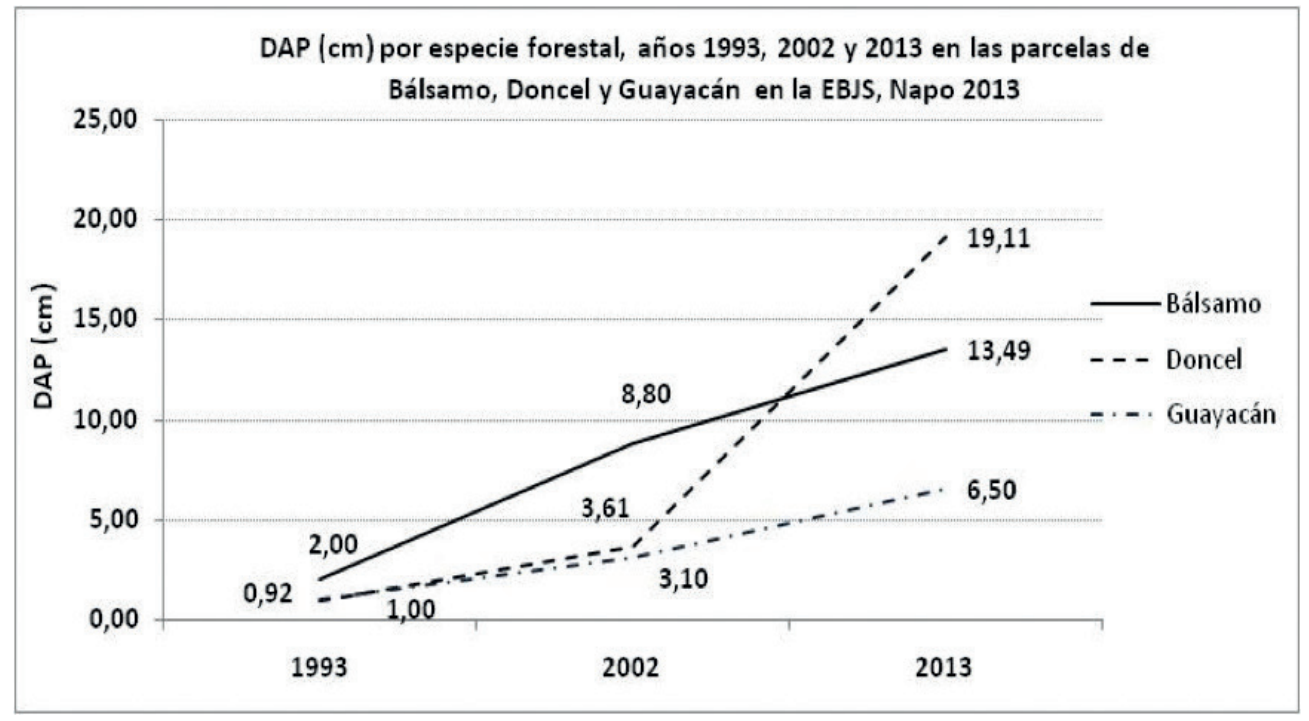

Figura 2. Diámetro a $1,30 \mathrm{~m}(\mathrm{DAP})$ promedio por especie forestal, años 1993, 2002 y 2013 a individuos en parcelas de Myroxylon balsamum (L.) Harms (Bálsamo), Minquartia guianensis Aubl. (Guayacán negro) y Otoba parvifolia (Doncel) establecidas en la Estación Biológica Jatun Sacha, Napo, 2013. 


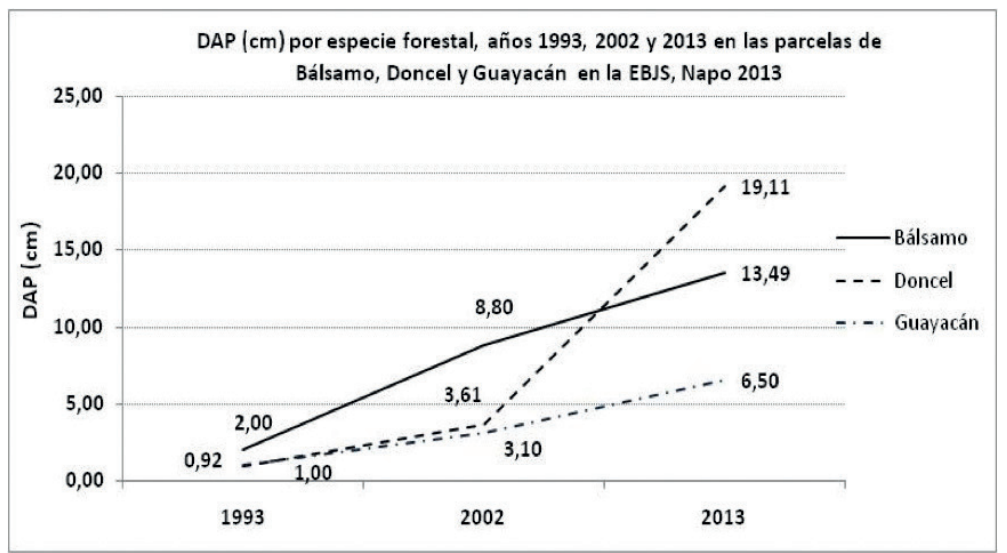

Figura 3. Alturas promedio por especie forestal, años 1993, 2002 y 2013 a individuos en parcelas de Myroxylon balsamum (L.) Harms (Bálsamo), Minquartia guianensis Aubl. (Guayacán negro) y Otoba parvifolia (Doncel) establecidas en la Estación Biológica Jatun Sacha, Napo, 2013.

En las figuras 2 y 3 encontramos los valores correspondientes a Diámetros y Alturas registrados en las tres especies analizadas en el periodo 1993 al 2013

\section{Ecuaciones alométricas}

Para las estimaciones de la tasa de crecimiento de las especies estudiadas, se utilizó los parámetros que detalla el anexo 3 del Acuerdo Ministerial 125 Ministerio del Ambiente, (MAE, 2015), que refieren que en la Región Amazónica y estribaciones el DMC de la especie Minquartia guianensis Aubl., es de 40 centimetros de diámetro; para la especie Myroxylon balsamum (L.) Harms es de 60 centimetros de diámetro y para la especie Otoba parvifolia es de 50 centímetros de diámetro. Considerando estos DMC, al realizar el cálculo de las ecuaciones alométricas obtuvimos un incremento por año y los años en que tardaría esta especie en alcanzar el Diámetro Mínimo de Corta DMC que está determinado por el Acuerdo
Ministerial 125 que derogó a la 039 vigente hasta febrero 2015, Ministerio del Ambiente (MAE, 2015).

Los resultados de las ecuaciones alométricas muestran que para la especie Myroxylon balsamum (L.) Harms se necesitan 96 años para obtener $60 \mathrm{~cm}$ de DAP, algo que debemos de tomar en consideración es que este análisis se realizo en una plantación de esta especie, la cual tuvo poco manejo, es decir se desarrollan en condiciones naturales. Para la especie Minquartia guianensis Aubl., de acuerdo a estas estimaciones, se necesitan 86 años para obtener $40 \mathrm{~cm}$ de DAP, algo que debemos de tomar en consideración es que este análisis se realizó en una plantación de esta especie, la cual tuvo poco manejo, es decir se desarrollan en condiciones naturales. Para la especie Otoba parvifolia se estimó que se requieren 27 años para obtener $50 \mathrm{~cm}$ de DAP, algo que debemos de tomar en consideración es que este análisis se realizó en una plantación de esta especie, la cual tuvo poco manejo, es decir se desarrollan en condiciones naturales (Ver figura 4).

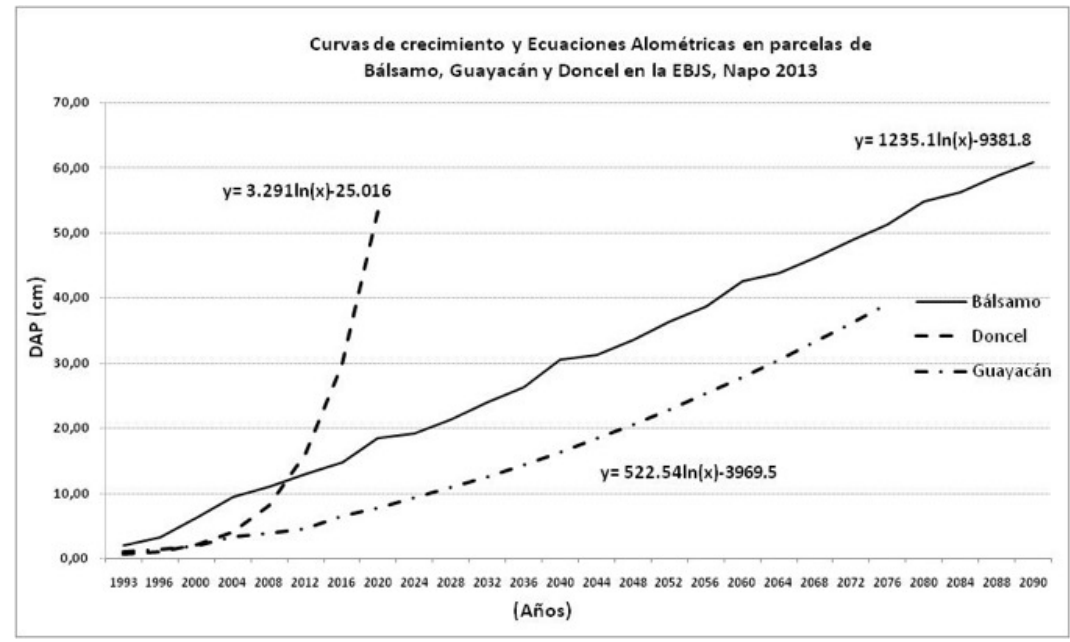

Figura 4. Ecuación Alométrica modelo de Chapman-Richards usada para calcular la tasa de crecimiento de Myroxylon balsamum (L.) Harms (Bálsamo), Minquartia guianensis Aubl. (Guayacán negro) y Otoba parvifolia (Doncel) en parcelas establecidas en la Estación Biológica Jatun Sacha, Napo, 2013. 


\section{Discusión}

$\mathrm{S}$ aprecia un incremento volumétrico moderado en las parcelas permanentes analizadas de las especies Bálsamo, Guayacán negro y Doncel; este estudio se realizó en el transcurso de 20 años, realizando 4 tomas de datos. En cambio Condit, Hubbel \& Foster (1995) estudio realizado en la Isla "Barro Colorado" realizaron el análisis de 6 especies forestales en el transcurso de 12 años, realizando cinco tomas de datos; en esta investigación al igual que -Condit et al., (1995)- se tomaron todos los diámetros existentes a una altura de 1,30 metros desde el nivel del suelo, obteniendo datos similares a este estudio.

Las especies Balsamo (Myroxylon balsamum (L.)) y Guayacán negro (Minquartia guianensis Aubl.), se desarrollaron muy lentamente en el lapso de tiempo analizado en "plantación pura", coincidiendo con (Somarriba et al., 2001) quienes encontraron en un estudio realizado en Costa Rica que el crecimiento en diámetro, altura y volumen del laurel fue 4.36 veces mayor en sistemas agroforestales que en plantaciones puras.

\section{Conclusión}

$\mathrm{E}$ crecimiento volumétrico del Balsamo y Guayacán negro es muy lento en comparación con el Doncel, de mantenerse esta tendencia sumado al aprovechamiento no controlado de estas especies, podría causar su extinción.

\section{Bibliografía}

Cerón, M. C. 1993a. Manual de botánica ecuatoriana, sistemática y métodos de estudio. Ediciones Abya Ayala. Quito, Ec. 315 p.

Cerón, C.E. \& E.L. Freire. 2005b. La vegetación y diversidad florística de Pavacachi, río Curaray, Pastaza - Ecuador. Cinchonia (Quito) 6(1): 14-28.

Condit, R., Hubbel S., \& Foster R. 1995, Demography and harvest potential of Latin American timber species: Data from a large, permanent plot in Barro Colorado island, Connecticut, USA

Gentry. A. 1996. A Field guide to the families and genera of woody the planta of northwest South America (Colombia, Ecuador, Perú), with supplementary notes on herbaceous txa. $895 \mathrm{p}$.

Guevara, J., Shiguango, H., \& Luna, D. 2009, Evaluación de la Flora arbórea de las comunidades alta Florencia, Río Napo y Bataburo, Río Tiwino; amazonía ecuatoriana. Cinchonia (Quito). 9 (1), Pp. 62-70.

Janzen, D.H. y C. Vasquez-Yanes. 1990. Aspects of tropical seed ecology of relevance to management of tropical forested wildlands. En A., Gomez-Pompa, T.C. Whitmore, y M. Hadley (eds.). Pp. 137-157. Rain Forest Regeneration and Management. UNESCO and Parthenon Publishing, Paris.

Jorgensen, P. \& León-Yánez, S. 1999. Catalogue of vascular plants of Ecuador. Missouri Botanical Garden Press. St.
Louis. U.S.A. 900 p.

Limogni, R. Guiracocha, G. 2012. Balsamo Myroxylon spp especie de uso múltiple del bosque seco de Ecuador. Estación Experimental Portoviejo, Estación Experimental del Litoral Sur. Programa Nacional de Forestería. Boletín técnico No 152. INIAP-MAGAP-SENESCYT. Editorial Grafiservi, Guayaquil, Ecuador. 20 p.

López, R. 2014a Tasas de Crecimiento de Especies Forestales y su Incidencia en la Dinámica del Mercado de Madera del Cantón Tena. Período 2007-2012. Propuesta De Aprovechamiento Sostenible. Tesis de Magister Scientiae en Manejo y Aprovechamiento Forestal. Ecuador

López, R. 2014b. El Bálsamo en Napo (Napo markapi tyak Bálsamo) Revista Socioambiental Huellas del Sumaco. Tena, Ecuador. 10: 6-9.

López, R. 2014c. El Guayacán negro - Huambula en Napo (Napo markapi tyak Guayacan negro - Huambula) Revista Socioambiental Huellas del Sumaco. Tena, Ecuador. 11: 6-10.

Melo, O. \& Vargas, R. (2002). Evaluación ecológica y silvicultural de ecosistemas boscosos. Universidad de Tolima. Ibagué, Colombia. 235 p.

Ministerio del Ambiente. MAE (2004). Acuerdo Ministerial 039. Normas para el Manejo Forestal Sostenible de los Bosques Humedos. Quito, Ec.

Ministerio del Ambiente. MAE (2009). Norma 139. De los procedimientos administrativos para autorizar el aprovechamiento y corta de madera. Quito, Ec.

Ministerio del Ambiente. MAE (2010). Aprovechamiento de los recursos forestales 2007 - 2009. Quito, Ec. 14 p.Ministerio del Ambiente, (2012): Estimación de la Tasa de Deforestación del Ecuador Continental, QuitoEcuador. 33 pp.

Ministerio del Ambiente. MAE (2015). Acuerdo Ministerial 125. Normas para el Manejo Forestal Sostenible de los Bosques Húmedos. Quito, Ec.

Neill, D.A., Palacios, W., Cerón, C.E., Mejía, L. 1993. Composition and Structure of Tropical Wet Forest in Amazonian Ecuador. En: Diversity and Edaphic Differentiation Association For Tropical Biology, Annual Meeting Pto. Rico.

Neill, D. 2012. ¿Cuántas especies nativas de plantas vasculares hay en Ecuador? Revista Amazónica Ciencia y Tecnología. Universidad Estatal Amazónica UEA, Puyo, Pastaza, Ec. 1: 70-83.

Palacios, W. 2011. Manual de identificación familias y géneros arbóreos del Ecuador. Quito, Ec. 123 p.

Pan William; S. J. Walsh; R. E. Bilsborrow; B. G. Frizzelle; C. M. Erlien; F. Baquero. 2004. Farm - level models of spatial patterns of land use and land cover dynamics in the Ecuadorian Amazon. Elsevier, Agriculture Ecosystem and Environment 101 (2004) 117 -134. Estados Unidos.

Pichon, F. and R. E. Bilsborrow 1999.Land-Use Systems, Deforestation and Associated Demographic Factors 
in the Humid Tropics: Farm - Level Evidence from Ecuador. Population and Deforestation in the Humid Tropics, edited by R. E. Bilsborrow and D. Hogan. Liege, IUSSP. Estados Unidos.Pretzsch, H., Utschig, H. and Sodtke, R. 2006. Applications of Tree Growth Modelling in Decision Support for Sustainable Forest Management. In Hasenauer, H. (Ed.) Sustainable Forest Management, Growth Models for Europe. Springer. Germany. 398 p.

Plumptre, A.J. 1995. The importance of "seed trees" for the natural regeneration of selectively logged tropical forest. Commonwealth Forestry Review 74:253-258.

Revelo, N. \& Palacios, W. 2005. Avances Silviculturales en la Amazonia ecuatoriana: Ensayos en la Estación Biologica Jatun Sacha, Fundacion Jatun Sacha \& Proyecto
CAIMAN. Quito. Ecuador. 172 pp.

Somarriba, E., Beer, J., Muschler, RG. 2001. Research methods for multistrata agroforestry system with coffee and cacao: Recommendations from two decades of research at CATIE, Agroforestry system 53(2): 195-203

Torres Bolier; L. Vallejo; J.P. Cedeño; A. Torres. 2013. La contribución del bosque amazónico a las condiciones de vida de la población rural pobre de la Reserva de Biosfera Sumaco, Ecuador. Revista Amazónica: Ciencia y Tecnología. ISSN 1390-5600

Vargas, B., Corral, J., Cruz, F., Aguirre, O., Nagel, J. 2008 Uso y aplicación de los simuladores de crecimiento forestal en la toma de decisiones silviculturales, Revista Forestal Latinoamericana, 23(2): 33-52. 